\title{
Reaction Wheel Disturbance Reduction Method Using Disturbance Measurement Table
}

\author{
Dong-Ik Cheon ${ }^{\dagger}$, Eun-Jeong Jang, and Hwa-Suk Oh \\ Department of Aerospace and Mechanical Engineering, Korea Aerospace University, Goyang 412-791, Korea
}

\begin{abstract}
Momentum changing actuators like reaction wheels and control moment gyros are generally used for spacecraft attitude control. This type of actuators produces force and torque disturbances. These disturbances must be reduced since they degrade the quality of spacecraft attitude control. Major disturbances are mainly due to static and dynamic imbalances. This paper gives attention to the reduction of the static and dynamic imbalance. Force/torque measurement system is used to measure the disturbance of the test reaction wheel. An identification method for the location and magnitude of the imbalance is suggested, and the corrections of the imbalance are performed using balancing method. Through balancing, the static and dynamic imbalance is remarkably reduced
\end{abstract}

Keywords: reaction wheel, disturbance, three-dimensional measurement table, imbalance, balancing

\section{INTRODUCTION}

Many types of actuators have been used for the satellite attitude control: magnetic torquer, momentum wheel, reaction wheel, control moment gyro and thrusters, etc. Actuators are selected according to the purpose of the satellite. Among them, momentum type actuators are often used for rotation accuracy and precise control.

A momentum type actuator is the instrument that stores angular momentum by means of a disk of large mass such as flywheel. The objective of a momentum actuator is to control and stabilize the satellite attitude on the basis of the law of conservation of angular momentum. This type of actuator is basically composed of a flywheel, motor, bearing, motor drive and controller. The advantage of a momentum type actuator is that it is appropriate to precise control since its composition is relatively simple and the control characteristics are good. The drawback is that the vibration generated by the rotator gives an adverse effect to the satellite control. Particularly, vibration is one of the important causes that bring about trembling in the imaging by the precise optical payloads
(Cheon et al. 2007).

The effects of disturbance should be reduced to carry out a more precise surveillance mission. Methods to reduce disturbance include reduction of generated vibration or attenuation of transmitted vibration. The former method, which is referred to as balancing, is to reduce the vibration of the rotator, while the latter method requires an additional attenuator at the reaction wheel. This paper is concentrated on the study of the former method.

Two different methods may be used to reduce the vibration generated by a rotor. The first one is to measure the roundness of the rotating wheel (or rotor) by measuring the shape of it or by attaching it to an axis of rotation and then measuring the rotational shape. This method is generally used when the rotator is composed of a single material, the shape is symmetric with reference to the axis of rotation, and when there is a surface of which shape can be easily measured. The second one is to use the vibration generated when rotating, which is called the disturbance. Sensors that can measure the vibration are attached to the structure that supports the axis of rotation, and the vibration signals are acquired by means of

(c) This is an Open Access article distributed under the terms of the Creative Commons Attribution Non-Commercial License (http://creativecommons.org/licenses/by-nc/3.0/) which permits unrestricted non-commercial use, distribution, and reproduction in any medium, provided the original work is properly cited.

Received Nov 01, 2011 Revised Nov 15, 2011 Accepted Nov 18, 2011 ${ }^{\dagger}$ Corresponding Author

E-mail: lionchun@kau.ac.kr

Tel: +82-2-3159-0147 Fax: +82-2-3158-3189 
the reflective tape and speed measurement sensor. The acquired data are analyzed to search the position and magnitude of the vibration source and to correct it. This method has no limitation and can be applied to various shapes of rotators, and is often used when the method of measuring the rotator shape is difficult to apply: from a small portable object to the screws for big container ships. The two methods are employed independently or in combination depending on the products of which vibration needs to be reduced.

However, reaction wheels have their own properties that make it hard to apply the methods described previously. Since a flywheel, which is the rotator inside the reaction wheel, should have great rotational inertia with respect to the weight, it is not a simple disk but a complex structure that needs to be fastened with the rotor of the actuator motor or the structure itself needs to play the role of a rotor (outer rotor type). Moreover, it is difficult to attach an outer sensor to the structure that supports the axis of rotation (case or housing), and the opening and closing of the case or housing are not convenient since the design considers the launching environment as well as the space environment.

More studies need to be thus conducted on balancing that considers such properties of reaction wheels. In this study, we suggested the balancing method to estimate the disturbance magnitude and the positions of disturbance factors. It is performed using a three-dimensional disturbance measurement table that enables to measure the disturbance. The z-phase detector detects the signals generated by rotation of the reaction wheel. The application of the method to a test reaction wheel resulted in the enormous reduction of disturbance.

\section{REACTION WHEEL DISTURBANCE MODELING}

The many factors that cause disturbance of momentum actuators, especially reaction wheels, include mass imbalance of the flywheel, bearings, irregular lubrication, ripple and cogging of the motor and the controller errors of the motor actuator, etc. Table 1 summarizes the types of disturbances depending on various vibration factors.

Among the vibration source factors in Table 1, the static imbalance and dynamic imbalance are related to the fundamental frequency and depends on the shape of wheel and distribution of the mass inside the wheel, as shown in Fig. 1 (Masterson et al. 1999).

The mathematical model is as follows:

$$
\begin{gathered}
F_{j}=\sum_{i=1}^{n} F_{j i} \Omega^{2} \sin \left(h_{i} \Omega t+\alpha_{j i}\right) \\
T_{j}=\sum_{i=1}^{n} T_{j i} \Omega^{2} \sin \left(h_{i} \Omega t+\alpha_{j i}\right)
\end{gathered}
$$

$F_{j}$ : the magnitude of the disturbance (force) on the $j$-th axis $T_{j}$ : the magnitude of the disturbance (torque) on the $j$-th axis $F_{j i}:$ the $i$-th mode parameter of $F_{j}$

$T_{j i}$ : the $i$-th mode parameter of $T_{j}$

$j: x, y, z$

$\Omega$ : the wheel rotation frequency

$h_{i}$ : the $j$-th harmonic number

$\alpha_{j i}$ : the $i$-th phase value of the $j$-th axis

Since harmonic number $h_{i}$ is a multiple of the wheel vibration speed, it can be expressed as follows:

$$
h_{i}=\frac{\omega_{i}}{\Omega}
$$

$h_{i}$ : the $i$-th harmonic number

$\omega_{i}$ : the $i$-th disturbance frequency

$\Omega$ : the wheel rotation frequency

Table 2 summarizes the relations among the parameters of the relevant forces, toques and the disturbance model on the basis of the modeling of the static and dynamic imbalances (Masterson et al. 2001).

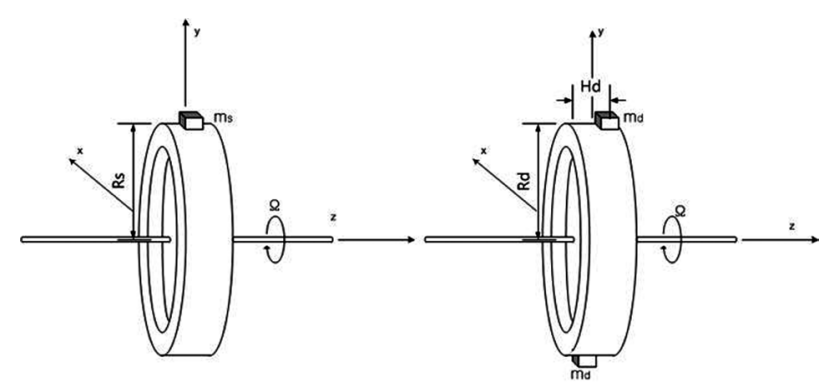

\begin{tabular}{|c|c|c|}
\hline $\begin{array}{l}\text { Disturbance } \\
\text { sources }\end{array}$ & Main causes & $\begin{array}{l}\text { Disturbances } \\
\text { type }\end{array}$ \\
\hline Wheel & Static imbalance & $\begin{array}{l}\text { Radial translational force } F_{x}, F_{y} \\
\text { Fundamental frequency }\end{array}$ \\
\hline Wheel & Dynamic imbalance & $\begin{array}{l}\text { Radial rocking torque } T_{x}, T_{y} \\
\text { Fundamental frequency }\end{array}$ \\
\hline Bearing & Lubricant irregularity & $\begin{array}{l}\text { Axial torque } T_{z} \\
\text { Sub and super harmonics }\end{array}$ \\
\hline Motor & Ripple cogging & $\begin{array}{l}\text { Axial torque } T_{z} \\
\text { High frequency }\end{array}$ \\
\hline Motor actuator & Controller error & $\begin{array}{l}\text { Axial torque } T_{z} \\
\text { Low frequency }\end{array}$ \\
\hline
\end{tabular}

Fig. 1. Static-dynamic disturbance model conception.

Table 1. The source and type of reaction wheel disturbance.

$x, y$ : radial direction, $z$ : axial. 
Table 2. Parameters of disturbance model.

\begin{tabular}{lll}
\hline \multicolumn{1}{c}{ Parameter } & \multicolumn{1}{c}{ Source } & \multicolumn{1}{c}{ Equation } \\
\hline Harmonic number & Amplitude spectrum & $H_{i}$ \\
Amplitude coef. & Amplitude spectrum & $F_{j}, T_{j}$ \\
Static imbalance & Radial force data & $F_{j i}=m_{s} R_{s}$ \\
& & $|F|=F_{j i} \Omega^{2}$ \\
Dynamic imbalance & Radial torque data & $T_{j i}=m_{d} R_{d} H_{d}$ \\
& & $|T|=T_{j i} \Omega^{2}$ \\
\hline
\end{tabular}

\section{THE TEST ACTUATOR AND THE DISTURBANCE MEASUREMENT SYSTEM}

To empirically verify the disturbance reduction, we employed a test reaction wheel, and the force/ torque disturbance measurement table shown in Fig. 2. The test actuator had the angular momentum of $1.94 \mathrm{Nms}$, the weight of $3.3 \mathrm{~kg}$, and the maximum revolution count of $3,000 \mathrm{rpm}$. The force, torque and disturbance measurement table allows the measurement of three-axis directional force and torque using four three-dimensional load cells. Table 3 shows the performance of the force/torque disturbance measurement table and its specifications when a wheel is installed. This system enables to measure the force and torque accurately by calibrating static and dynamic loads (Gong et al. 2010a,b).

\section{REDUCTION OF THE STATIC AND DYNAMIC IMBALANCE DISTURBANCE}

\subsection{Identification of Imbalances and Their Reduction}

As shown in Fig. 1, the wheel disturbances are modeled by classifying the causes of disturbance into static and dynamic mass imbalances. As the mass imbalance of the disk wheel generates centrifugal force by rotation, the static mass imbalance, $m_{s}$, gives force. The two dynamic mass imbalances, $m_{d}$, cause disturbance torque as the centrifugal force generated by rotation turns to a couple of forces, since they are separated by the distance of $H_{d}$ (Masterson et al. 2001). As shown in Fig. 2, the test actuator having imbalance mass was run on the force/torque measurement table, and the disturbance generated by rotation was measured. The relationship between the imbalance mass of the test actuator and the measurement table can be defined as in Fig. 3 to estimate the positions of the imbalance masses.

Fig. 3 is the schematic diagram that shows how to find the force and the torque vector $\vec{D}$ that causes the static- dynamic disturbance. In Fig. 3, x-y denotes the axes fixed on the wheel, while $\mathrm{X}-\mathrm{Y}$ denotes the axes fixed on the table. The imbalance mass that generates $\vec{D}$ is referred to as the equivalent imbalance mass, and it was marked as 'static' or 'dynamic,' according to the mass. But, the static and dynamic vectors $\vec{D}$, are independent with each other. After estimating the direction and magnitude of the individual $\vec{D}$ vectors in Fig. 3, the position and magnitude of the actual imbalance masses can be derived. The

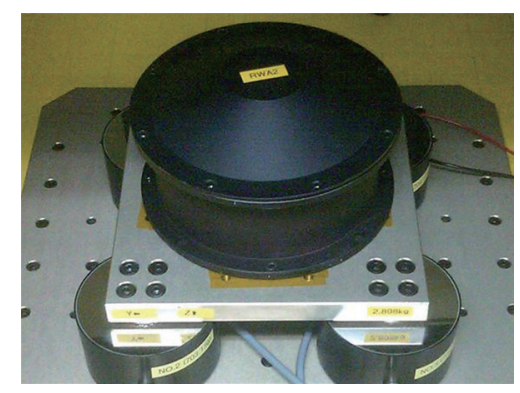

Fig. 2. Disturbance measurement table and standard actuator.

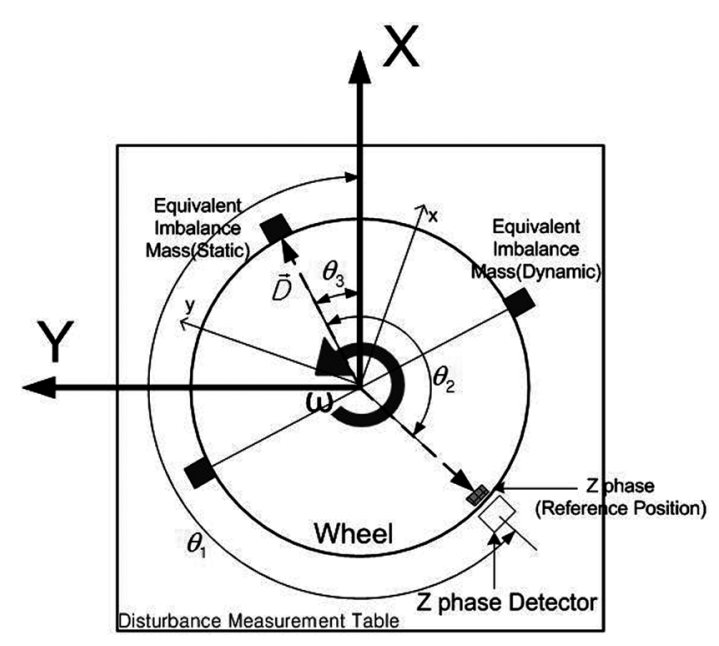

Fig. 3. Conception of disturbance vector $\mathrm{D}$ detection.

Table 3. Disturbance measurement table specification.

\begin{tabular}{|c|c|c|c|c|c|c|}
\hline & Fx & Fy & $\mathrm{Fz}$ & $\mathbf{T x}$ & Ty & $\mathrm{Tz}$ \\
\hline Range (N, N-m) & $\pm 1,200$ & $\pm 1,200$ & $\pm 1,200$ & 108 & 108 & 216 \\
\hline Natural frequency $(\mathrm{Hz})$ & 415 & 456 & 692 & 1,057 & 1,039 & 673 \\
\hline $\begin{array}{l}\text { Natural frequency } \\
\text { with wheel }(\mathrm{Hz})\end{array}$ & 284 & 312 & 473 & 689 & 677 & 535 \\
\hline Linearity & \multicolumn{6}{|c|}{$0.3 \%$ of R.O } \\
\hline Hysteresis & \multicolumn{6}{|c|}{$0.3 \%$ of R.O } \\
\hline Repeatability & \multicolumn{6}{|c|}{$0.1 \%$ of R.O } \\
\hline Overload & \multicolumn{6}{|c|}{ $\pm 120 \%$ R.C } \\
\hline Noise $(\mathrm{N} / \sqrt{ } \mathrm{Hz}, \mathrm{N}-\mathrm{m} / \sqrt{ } \mathrm{Hz})$ & \multicolumn{6}{|c|}{$<0.028$} \\
\hline Resolution & \multicolumn{6}{|c|}{16 bit } \\
\hline
\end{tabular}

R.O: Rated Output, R.C: Rated Capacity 
position and magnitude of static imbalance mass and the equivalent imbalance mass are the same. The dynamic imbalance mass has the same magnitude but is located at $\pm 90^{\circ}$ of $\vec{D}$.

The force equation can be summarized as in Eq. (3) using the first harmonic number corresponding to the fundamental frequency in the previous modeling equation:

$$
F_{j}=F_{j 1} \Omega^{2} \sin \left(h_{1} \Omega t+\alpha_{j 1}\right)
$$

The equation can be rearranged with reference to the $\mathrm{X}$ axis of the table:

$$
F_{x}=F_{x 1} \Omega^{2} \sin \left(h_{1} \Omega t+\alpha_{x 1}\right)
$$

A position signal can serve as a cross reference for the wheel and the table, and the $z$ phase can play the role. The $\mathrm{z}$ phase is the signal that gives one pulse per revolution, and it is generated in connection with the rotating wheel. When the wheel is driven, the signal waveform of the force or torque measured by the reference axis of the disturbance measurement table can be acquired as the $\mathrm{z}$ phase of the wheel passes through the $\mathrm{z}$ phase detector. Since the signal has a periodic characteristic, the phase value can be extracted through Fast Fourier transform.

Here, the phase value is $\alpha_{x 1}$, and the position of the equivalent imbalance mass is at the position angle of $\theta_{3}$, and thus the for or torque generated by the equivalent imbalance mass is as follows:

$$
D_{x}=D_{i m} \cos \left(\theta_{3}\right)
$$

By equating (5) with (4), we obtain

$$
F_{x}=D_{x}, \quad F_{x 1} \Omega^{2}=D_{i m}
$$

The same result is obtained by applying torque. Setting $\mathrm{t}=0$ at the time when passing through the $\mathrm{z}$ phase detector later gives

$$
\sin \left(a_{x 1}\right)=\cos \left(\theta_{3}\right)
$$

The condition to satisfy Eq. (7) is

$$
\alpha_{x 1}=2 n \pi+\theta_{3}-\pi / 2, \quad n=0,1,2 \cdots
$$

The phase, the angle of the imbalance mass with respect to the reference detector, should be within $2 \pi$. Since $n=1$, rearrangement of the equation with respect to $\theta_{3}$ gives,

$$
\theta_{3}=\alpha_{x 1}+\pi / 2
$$

The position angle of the imbalance mass from the $\mathrm{z}$ phase is

$$
\theta_{2}=2 \pi-\left(\theta_{1}-\theta_{3}\right)
$$

To cancel off the imbalance mass at the position, the wheel disturbance is reduced by attaching a counter mass corresponding to the imbalance masses of $m_{s}$ and $m_{d}$, as shown in Fig. 4.

\subsection{Balancing Process}

In the balance process, there exist two experimental constrains due to the structural factors of the test actuator itself.

First, with respect to the force and torque generated by the disturbance, $F_{x}$ and $F_{y}$, and $T_{x}$ and $T_{y}$ should be measured in the same manner by the disturbance modeling. However, due to the structural factors of the brushless direct current motor of the test reaction wheel employed in this study, the characteristic frequency is low in y-axis rotational direction, and thus the balancing process was performed with reference to the measurements of $F_{y}$ and $T_{x}$. Second, the process was performed with reference to the revolution count as low as $600 \mathrm{rpm}$ in order not to measure the twisted disturbance values since the characteristic frequency of the internal structure of the test actuator was low.

In the balancing process, the static imbalance was firstly reduced and then the dynamic imbalance was reduced. The data for the static imbalance was acquired in the time domain with reference to the $\mathrm{z}$ phase after starting to keep the test actuator at a constant rate of $600 \mathrm{rpm}$. The position of the imbalance mass was found by the method suggested in Fig. 3. The test actuator was re-driven after attaching the counter mass as shown in Fig. 4. To attach the counter mass, we applied small set screws or blots to the holes tapped on the height of $H_{d}$ in $30^{\circ}$ interval for the disturbance correction in the reaction wheel fabrication.

It showed that the magnitude of the disturbance was reduced. The disturbance is reduced step by step by applying the abovementioned method iteratively. As shown in Fig. 5, however, the phase of the disturbance is difficult

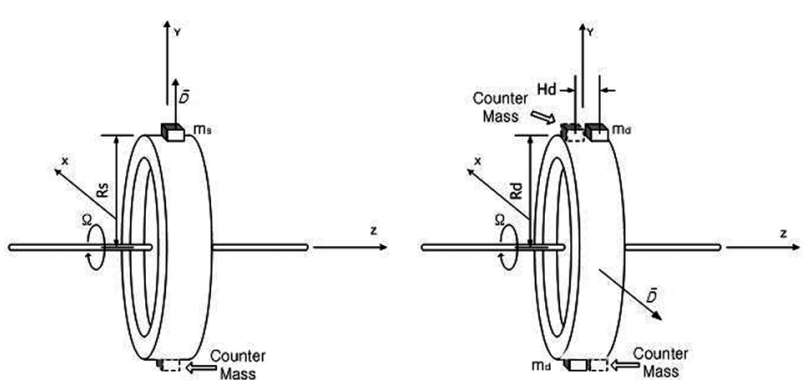

Fig. 4. Balancing conception (addition counter mass). 
to decide visually with the data acquired in the time domain. The magnitude and phase of the disturbance can be easily identified with a lower pass filter so that the frequency corresponding to the first harmonic number can be found as shown in Fig. 6. However, the phase delay caused by the application of a low pass filter should be compensated later.

In summary, the first stage corresponds to the Figs. 5 and 6 , the second stage to the Figs. 7 and 8 , and the third stage to the Figs. 9 and 10. As the balancing process is applied to the dynamic imbalance, Figs. 11 and 12 show the dynamic imbalance after the static imbalance balancing.

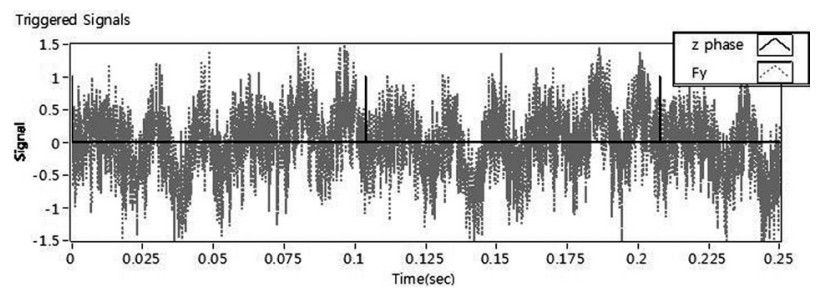

Fig. 5. Force disturbance on time domain-Step 1.

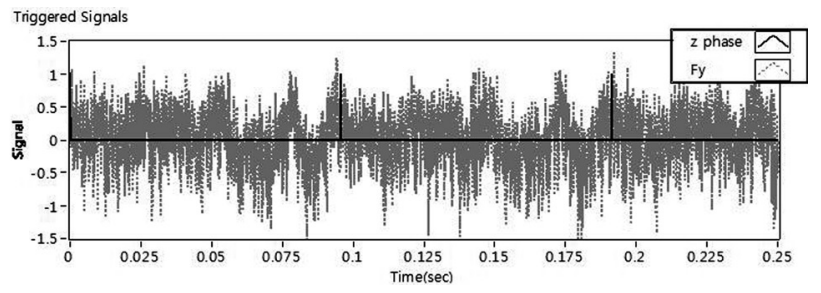

Fig. 7. Force disturbance on time domain -Step 2.

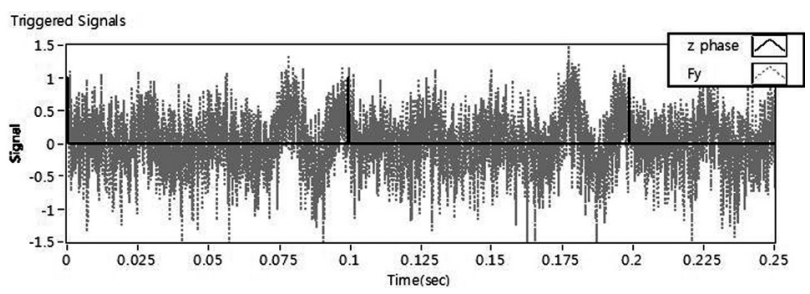

Fig. 9. Force disturbance on time domain -Step 3.

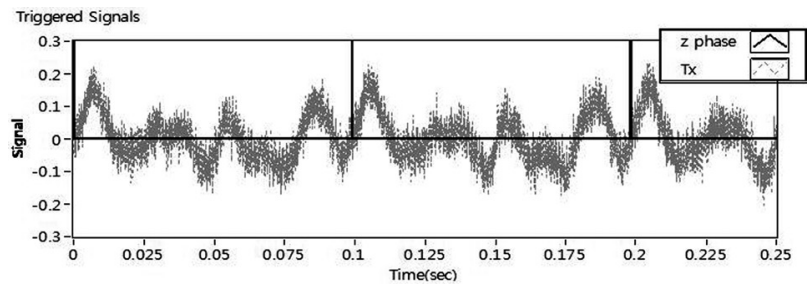

Fig. 11. Torque disturbance on time domain-Step 1.
Figs. 13 and 14 show the result of the application. Fig. 15 is the flow diagram of the balancing process.

Table 4 shows the measurements in the static balancing process, and Table 5 are the values shown in the dy-

Table 4. Result table of static balancing process.

\begin{tabular}{cccccc}
\hline $\begin{array}{c}\text { Process } \\
\text { step }\end{array}$ & $\begin{array}{c}\text { Static } \\
\text { balancing } \\
\text { mass }\end{array}$ & $\begin{array}{c}\text { Dummy } \\
\text { mass angle }\end{array}$ & $\begin{array}{c}\text { g cm } \\
(@ 600 \text { rpm })\end{array}$ & $\begin{array}{c}\text { g } \\
\text { (req) }\end{array}$ & $\begin{array}{c}\text { Detect } \\
\text { angle }\end{array}$ \\
\hline 1 & & & 6.328 & 1.11 & -93.242 \\
2 & $1.3 \mathrm{~g}$ & -90 & 2.362 & 0.414 & 121.943 \\
3 & $0.4 \mathrm{~g}$ & 120 & 1.276 & 0.22 & 219.342 \\
\hline
\end{tabular}

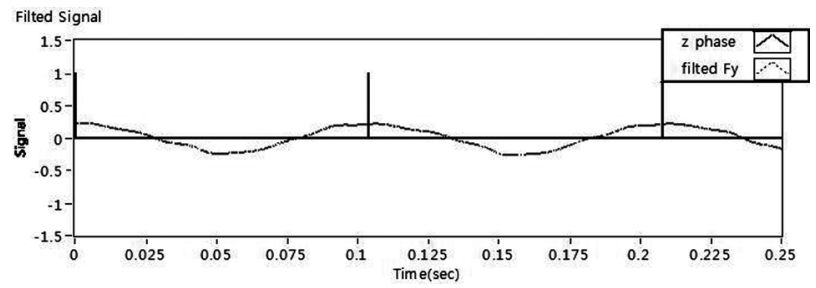

Fig. 6. Force disturbance on time domain -Step 1 (low pass filter).

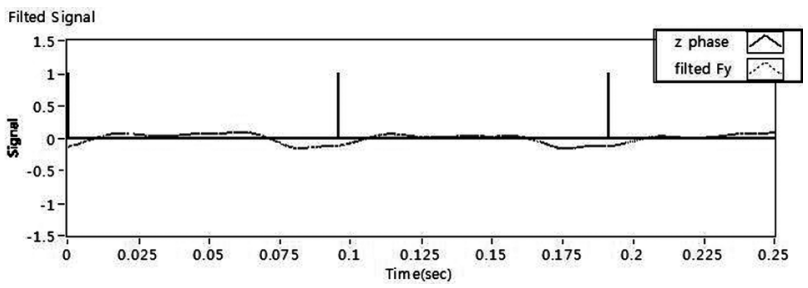

Fig. 8. Force disturbance on time domain -Step 2 (low pass filter).

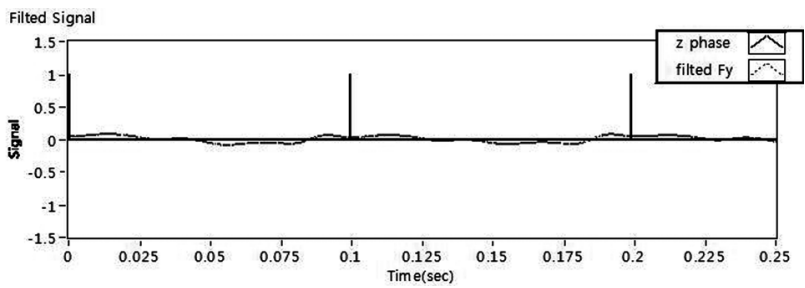

Fig. 10. Force disturbance on time domain -Step 3 (low pass filter).

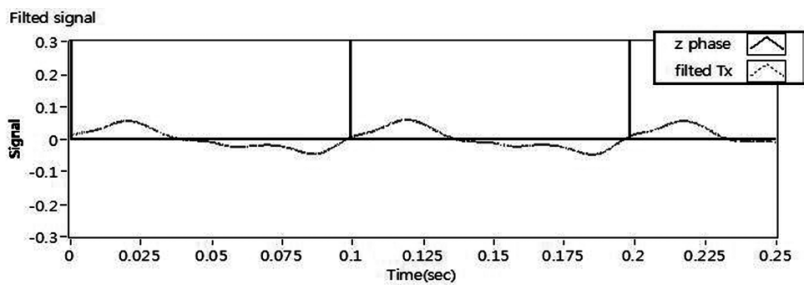

Fig. 12. Torque disturbance on time domain -Step 1 (low pass filter). 


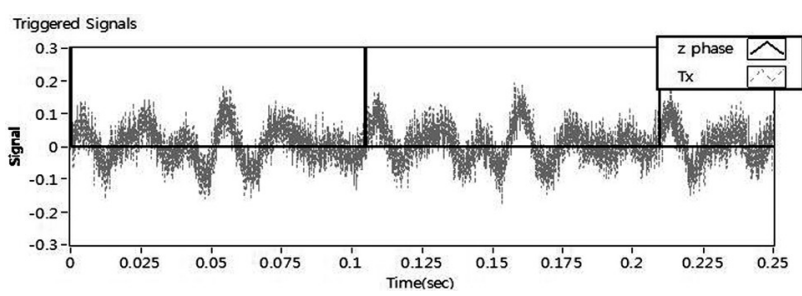

Fig. 13. Torque disturbance on time domain -Step 2.

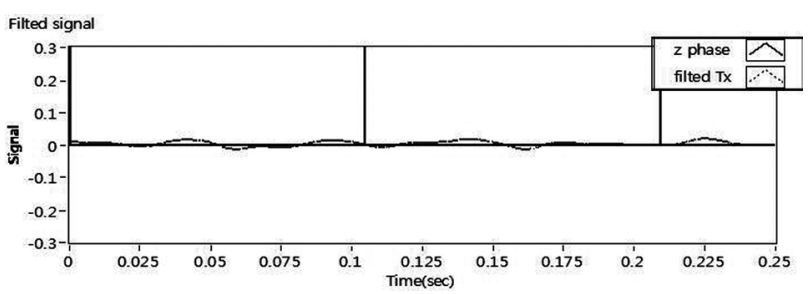

Fig. 14. Torque disturbance on time domain -Step 2 (low pass filter).

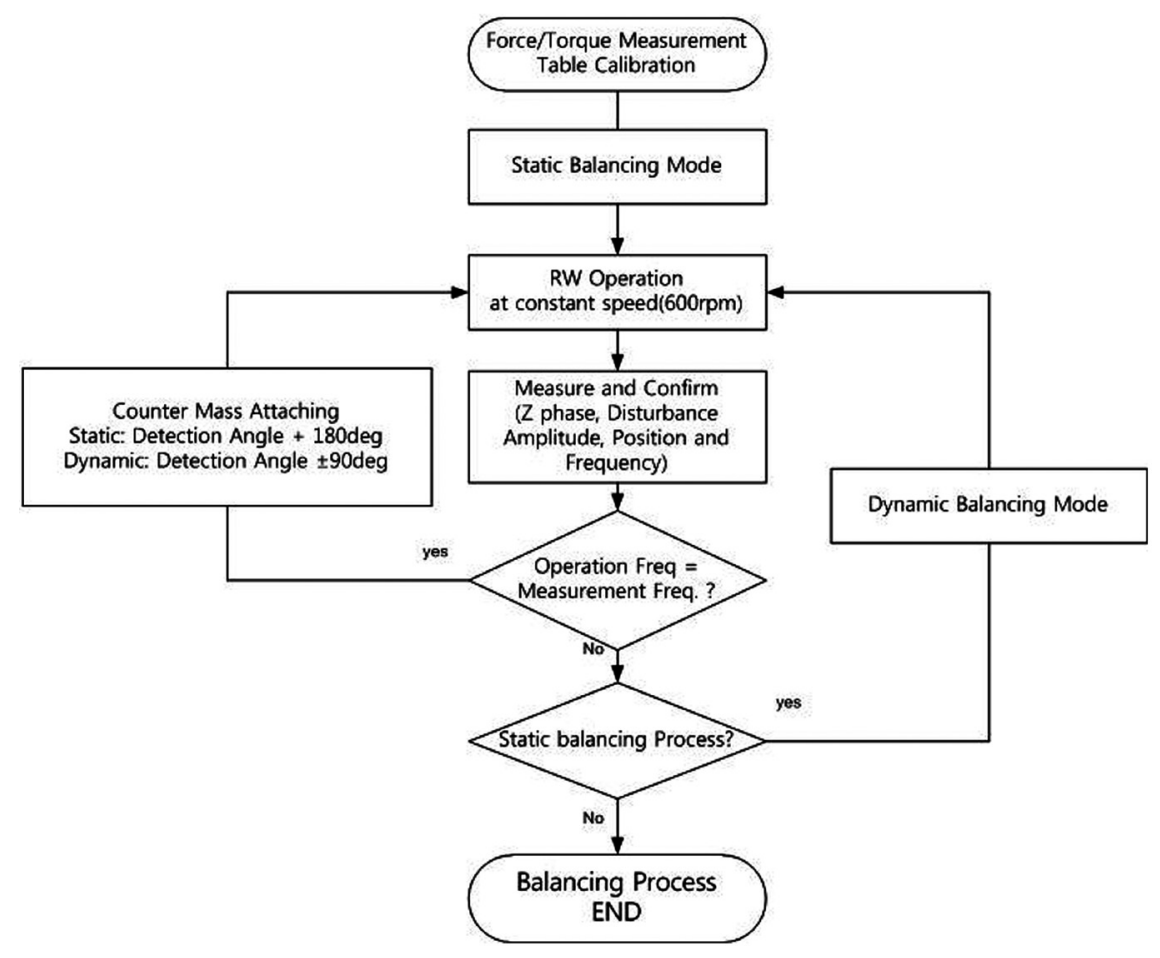

Fig. 15. Balancing process flow diagram.

namic balancing process. In Tables 4 and $5, g_{\text {req }}$ denotes the required counter mass, and the balancing mass is the attached counter mass. The detect angle $\theta_{3}$ is the position of the imbalance mass estimated through the disturbance experiment. The angle denotes to the angle of the counter mass, which is $\left(\theta_{3}+\pi\right)$. The $g_{\text {req }}$ in step 1 is $10.518 \mathrm{~g}$ in Table 5 , but we applied the maximum attachable counter mass of mass $5.4 \mathrm{~g}$ to the reaction wheel in step 2 .

Table 5. Result table of dynamic balancing process.

\begin{tabular}{cccccc}
\hline Step & $\begin{array}{c}\text { Balancing } \\
\text { mass }\end{array}$ & Angle & $\begin{array}{c}\mathbf{g ~ c m}^{2} \\
(@ 600 ~ \mathbf{~ r m})\end{array}$ & $\begin{array}{c}\mathrm{g} \\
(\text { req })\end{array}$ & $\begin{array}{c}\text { Detect } \\
\text { angle }\end{array}$ \\
\hline 1 & & & 110.836 & 10.518 & -120.974 \\
2 & $5.4 \mathrm{~g}(\max )$ & -120 & 9.78 & 0.85 & -82 \\
\hline
\end{tabular}

\section{RESULTS AND DISCUSSION}

The balancing processes are performed to reduce the disturbance by repeating the suggested method with regard to the static-dynamic imbalance. The experimental result showed that the disturbance was reduced. Table 6 summarizes the result for the static and dynamic disturbances by presenting the separated results together.

In the individual stages of the static balancing process shown in Table 6, the magnitude of the disturbance was reduced from 6.328 to 1.276 , while the dynamic imbalance did not show a reducing trend since the figure was changed from 136.484 to 97.113 and then to 110.836 . This result showed that interference was generated by the complex structure and vibration of the test actuator and 
Table 6. Overall result table of balancing process.

\begin{tabular}{|c|c|c|c|c|c|c|c|c|c|c|c|c|}
\hline $\begin{array}{l}\text { Balancing } \\
\text { step }\end{array}$ & $\begin{array}{l}\text { Static } \\
\text { step }\end{array}$ & $\begin{array}{c}\text { Static } \\
\text { balancing } \\
\text { mass }\end{array}$ & $\begin{array}{c}\text { Dummy } \\
\text { mass } \\
\text { angle }\end{array}$ & $g(r e q)$ & $\mathrm{g} \mathrm{cm}$ & $\begin{array}{l}\text { Detect } \\
\text { angle }\end{array}$ & $\begin{array}{c}\text { Dynamic } \\
\text { step }\end{array}$ & $\begin{array}{c}\text { Dynamic } \\
\text { balancing } \\
\text { mass }\end{array}$ & $\begin{array}{c}\text { Dummy } \\
\text { mass } \\
\text { angle }\end{array}$ & $g(r e q)$ & $\mathrm{g} \mathrm{cm}^{2}$ & $\begin{array}{c}\text { Detect } \\
\text { angle }\end{array}$ \\
\hline 1 & 1 & & & 1.11 & 6.328 & -93.2421 & & & & 12.943 & 136.484 & -232.172 \\
\hline 2 & 2 & $1.3 \mathrm{~g}$ & -90 & 0.414 & 2.362 & 121.943 & & & & 9.2094 & 97.113 & -102.962 \\
\hline 3 & 3 & $0.4 \mathrm{~g}$ & 120 & 0.22 & 1.276 & 219.342 & & & & 10.518 & 110.836 & -120.974 \\
\hline 4 & & & & 0.178 & 1.018 & -111 & 1 & $5.4 \mathrm{~g}(\max )$ & -120 & 0.85 & 9.78 & -82 \\
\hline
\end{tabular}

the disturbance measurement table. But the static and dynamic imbalance modeling in Fig. 1 and the balancing concept in Fig. 4 are still valid. However, the dynamic imbalance of the test actuator exceeded the maximum allowable counter mass for the dynamic balancing and thus the dynamic disturbance could not be further reduced. A countermeasure needs to be taken for the structure of the test actuator in the future.

\section{CONCLUSIONS}

Momentum type actuators that used for satellite attitude control are widely used since they provide rotational stability and precise control performance. However, the centrifugal force and their couple caused by wheel rotation generate disturbance and results in the vibration. The vibration is one of the causes that decrease the attitude control performance of a satellite that requires accurate orientation as well as the performance of the precise payloads such as a high-resolution camera. For this reason, various studies are conducted to reduce the disturbance.

To reduce the disturbance, we performed modeling of the disturbance and estimated the position of imbalance mass. The disturbances are measured by a three-dimensional disturbance measurement table that allows precise measurement of the disturbance. Firstly, we performed the balancing process to reduce the static imbalance, which is the dominant disturbance, and then repeated the same process to reduce the dynamic imbalance. The result showed that the disturbance was reduced and that the suggested method for the disturbance modeling was effective.

The result of this study may help the balancing study to reduce the vibration of not only momentum actuators but also other rotators and rotating mechanical elements that require precise control. The performance of the three-dimensional disturbance measurement table needs to be improved further for the more accurate measurement.

\section{ACKNOWLEDGEMENTS}

This study was supported by the National Research Fund of Korea (No. 2011-0029878) funded by the 2011 budget of the Korean government (Ministry of Education, Science and Technology) and by the Global Surveillance Research Center at the Agency for Defense Development.

\section{REFERENCES}

Cheon D-I, Lee H-H, Oh H-S, Three dimensional measurements and parameter identification of force and torque disturbances of high speed rotating actuators, J Korean Soc Mech Eng, 31, 409-416 (2007).

Gong SC, Cheon D-I, Oh H-S, Calibration method of measurement table for precision disturbance measurement, in 2010 Korean Space Science Society Fall Conference, Pyeongchang, Korea, Oct 2010a, 35.

Gong SC, Kim JH, Kim J-C, Cheon D-I, Oh H-S, Conceptual design of micro-disturbance measurement table, in 2010 Society for Aerospace System Engineering Spring Conference, Goyang, Korea, Jun 2010b, 62-67.

Masterson RA, Miller DW, Grogan RL, Development and empirical and analytical reaction wheel disturbance models, in Proceedings of the 40th AIAA/ASME/ASCE/ AHS/ASC Structures, Structural Dynamics, and Materials Conference and AIAA/ASME/AHS Adaptive Structures Forum and AIAA Non-Deterministic Approaches Forum, St. Louis, MO, 12-15 Apr 1999, AIAA paper A9924904.

Masterson RA, Miller DW, Grogan RL, Development and validation of reaction wheel disturbance models: empirical model, J Sound Vib, 249, 575-598 (2001). http://dx.doi. org/10.1006/jsvi.2001.3868 